\title{
Einhörner und Geschichtsunterricht?!
}

\section{Geschichtsdidaktischer Kommentar ${ }^{1}$}

Über die Rolle des Einhorns als ein für uns zuverlässig fiktionales Wesen für Geschichtsdidaktik und -unterricht zu diskutieren, mag zunächst merkwürdig und alles andere als wissenschaftlich erscheinen, werden mit Einhörnern doch mehr „New-Age-Schwärmereien“ oder „verschwommene[s], konturlose[s], magische[s] Zauberwesen“2 assoziiert. Unsere moderne Lebenswelt ist ohnehin voll von Einhörnern als mystische und schöne Fabelwesen in Form von Textildrucken, Schlüsselanhängern oder sogar Nahrungsmitteln wie Pasta in Einhornform. Folglich dürfte die Beschäftigung mit Einhörnern für viele auf einer Stufe mit ,HelloKitty“ und Co stehen. Allerdings wird schon beim tieferen Nachdenken über den Gegenstand ,Einhorn` klar, dass unsere Vorstellungen immer abhängig von unserem Hintergrund sind - denn die Beschäftigung mit dem Einhorn ist nun eben kein modernes Kitsch- oder Kinder-Phänomen, sondern schon seit jeher, wie schon in antiken Abhandlungen von Gelehrten deutlich wird, existent gewesen. Erwähnt sei an dieser Stelle beispielsweise die Schrift Indika des Ktesias von Knaidos von etwa 298 v. Chr., in der eine Geschichte eines Tieres mit einem einzigen Horn erzählt wird - eine Legende, „die in der Vorstellungswelt des Abendlandes mehr als 2.000 Jahre überdauert hat“. ${ }^{3}$ Vor diesem Hintergrund wirft Julia Weitbrecht in ihrem Vortrag ,Mensch-Tier-Beziehungen in historischer Perspektive: Zur Faszinationsgeschichte des Einhorns' auch geschichtsdidaktische Schlaglichter auf die Vormoderne. Doch kann ein „Tier, das es nicht gibt““, wie Rilke in Die Sonette an Orpheus treffend schreibt, wirklich Gegenstand eines Geschichtsunterrichts und einer Geschichtsdidaktik sein, in deren Zentrum auch die Historizität als solche steht?

\section{Tiere als Teil der (mittelalterlichen) Lebenswelt}

In ihrem Beitrag stellt Julia Weitbrecht zunächst die Relevanz der Beschäftigung mit Tieren allgemein heraus, die einen großen Teil der mittelalterlichen Lebenswelt ein-

\footnotetext{
1 Kommentierter Beitrag: https://dx.doi.org/10.38072/2703-0784/p8.

2 Chris Lavers, Das Einhorn. Natur, Mythos, Geschichte, Darmstadt 2010, 7.

3 Ebd., 9.

4 Rainer Maria Rilke, Die Sonette an Orpheus, Zweiter Teil, IV.
}

\footnotetext{
>> Das Werk ist unter der Creative-Commons-Lizenz Namensnennung - Weitergabe unter gleichen Bedingungen 4.0 International veröffentlicht. Den Vertragstext finden Sie unter: https://creativecommons.org/licenses/by-sa/4.0/deed.de. Bitte beachten Sie, dass einzelne, entsprechend gekennzeichnete Teile des Werks von der genannten Lizenz ausgenommen sein bzw. anderen urheberrechtlichen Bedingungen unterliegen können.
} 
nehmen. Tiere eignen sich, auch heute noch, wirft man einen Blick in Märchen, Fabeln, Disney-Filme u.s.w., um Themen jeglicher Art zu veranschaulichen oder Wissen zu transportieren. So werden Tiere auch in der Vormoderne funktionalisiert und produktiv genutzt, indem man sie etwa selbst erzählen lässt. Dass bestimmte Tiere stilisiert werden, z. B. auch, indem ihnen verschiedene Eigenschaften zugesprochen werden, ist kulturell gewachsen und hat ihren wesentlichen Ursprung im Mittelalter. So stellt die Verknüpfung des Fuchses mit Listigkeit eine Kontinuität dar, die den meisten nicht fern sein dürfte. Weitbrecht sieht auf dieser Basis Tiere als Medien der Spiegelung und Reflexion, die aber auch auf die Grenzen der Menschen und ihrer Menschlichkeit hinweisen.

Die Menschen griffen dafür auf unterschiedliche Wissensbestände - z. B. Religion und Naturkunde, die in der Vormoderne keineswegs widersprüchlich zu sein scheinen - zurück, wodurch sich ein sehr weiter Literaturbegriff ergibt. Das Korpus der Tiergeschichten ist demnach nicht erkennbar systematisch, denn es "reiht reale und phantastische, heimische und fremde, bekannte und unbekannte Wesen aneinander. “5 Neben der Bibel ist wohl der Physiologus als wichtiger Text für die Beschäftigung mit Tieren im Mittelalter zu nennen, der nicht nur im mittelalterlichen Europa weit verbreitet, sondern auch Vorlage für die mittelalterlichen Bestarien war. ${ }^{6}$ Darüber hinaus sind noch die Fabeltradition und mittelalterliche Epen zu nennen, in denen Tieren eine wesentliche Rolle zukommt. Reinhart Fuchs ist ein solches Tierepos, das auch heute noch im kollektiven Gedächtnis verankert ist. Insgesamt werden etwaige Informationen über Tiere im Mittelalter und ihre Beziehungen zum Menschen also aus verschiedenen Wissensbeständen, sprich aus naturkundlichen, literarischen und religiösen, generiert.

Schon an dieser Stelle ließen sich fachdidaktische Überlegungen anstellen, zeigt sich doch eindrücklich, was sich durch mittelalterliche Tierkunde alles historisch ableiten lässt. Wo Tiere Teil und Spiegel der mittelalterlichen Lebenswelt sind, ergibt sich ein großer didaktischer Nutzen. Prinzipien des quellenbasierten, forschend-entdeckenden Lernen greifen hier, wenn Schülerinnen und Schüler anhand der genannten Schriften Tiere als „Symbole und als Mittel zur ,Zurichtung“ von Menschen“7 betrachten. Der weit gefasste Literaturbegriff kann hier zum Vorteil gereichen, bieten sich doch verschiedene Textsorten und damit eine größere Variation zur Untersuchung an. Besonders viel Potenzial sehe ich allerdings für die Ausbildung des Geschichtsbewusstseins, welches mit dem Schwinden von Normativen in der geschichtsdidaktischen Diskussion im Fokus des Interesses steht. Diesbezüglich führt Ammerer an, das Geschichtsbewusstsein beginne dort, „wo Zusammenhänge (z. B. Entwicklungen, Kausalitäten, Kontinuitäten, Brüche,...) erkannt werden zwischen (der Erinnerung an die) Vergangenheit, (der Deutung

5 Ingrid Matschinegg, Tierkunde im Mittelalter. Tiere im Interessensfeld zwischen christlicher Heilslehre und profaner Wissenschaft, in: Historische Sozialkunde 41, 2011, 4, 4-10, hier 4.

6 Vgl. ebd., $4 \mathrm{f}$.

7 Thomas Hellmuth, Funktion und Wandel von „Tierbildern". Didaktische Variationen zu einem ungewöhnlichen Thema, in: Historische Sozialkunde (wie Anm. 5), 37-47, hier 37. 
von) Gegenwart und (der Erwartung an die) Zukunft“ und entfalte sich dort, „wo sich das Subjekt selbst in Bezug zu diesen drei Ebenen setzen kann “. ${ }^{8}$ Diese Denkkategorie wird Schülerinnen und Schülern natürlich nicht automatisch in die Wiege gelegt, was für uns als Geschichtsdidaktiker*innen bedeutet, dass historisch zu denken erst einmal gelehrt und gelernt werden muss.

\section{Das Einhorn in der Vormoderne: Vom Symbol der Christlichkeit bis zum ,Alicorn'}

Ebendies sollen zunächst nur einzelne Schlaglichter der geschichtsdidaktischen Perspektive sein, die allerdings bereits vielversprechend erscheinen. Doch können diese auch für die historische Auseinandersetzung mit einem gänzlich fiktiven Wesen, wie wir heute wissen, gelten? Erst jetzt kommt Julia Weitbrecht zu ihren Ausführungen speziell über das Einhorn. Dieses sei in ihrer Ursprünglichkeit immer mit einer gewissen ,Wildheit‘ assoziiert worden, die hier auch eine ,Ungezähmtheit‘ meint. In ihrem Beitrag wirft sie an dieser Stelle ein, dass sich diese Assoziation anböte, um mit denen der Schülerinnen und Schüler verglichen zu werden. Schüler*innenvorstellungen (als Einstieg in eine Einheit) zu generieren, weckt nicht nur das Interesse der Lerngruppe, sondern zeigt ihnen auch einen direkten Lebensweltbezug und damit eine Relevanz des behandelten Gegenstands. Solche Vorstellungen sind daneben auch immer in die Geschichtskultur ${ }^{9}$ eingebettet, wie einleitend bereits deutlich wurde. Um durch Assoziationen Verknüpfungen oder Kenntnisse der Schülerinnen und Schüler zu ermitteln, gibt es beispielsweise die Möglichkeiten eines Brainstormings oder Schreibgesprächs, wie Adamski und Bernhard anführen. ${ }^{10}$

Im Merkmal der ,Wildheit' geeint, wird das Einhorn in der vormodernen Vorstellungswelt insgesamt, wie es Weitbrecht schon für die mittelalterliche Tierliteratur generell festgemacht hat, mittels verschiedener Sujets verhandelt. So ist auch das Einhorn Gegenstand der Naturlehre: „Die Orsäer in Indien jagen [...] als wildestes Tier aber das Einhorn, das sonst am Körper dem Pferde, am Kopf aber dem Hirsch, an den Beinen dem Elefanten, am Schwanz dem Eber ähnlich ist, dumpf brüllt, während ein zwei Ellen langes schwarzes Horn mitten auf der Stirne hervorragt. " ${ }^{11}$ Lebend soll sich dieses Wild nicht fangen lassen, schreibt bereits Plinius in seiner Naturalis Historia, in dessen Passage der Aspekt der bereits angesprochenen Wildheit nochmals deutlich

\footnotetext{
8 Heinrich Ammerer, Geschichtsbewusstsein als grundlegende Kategorie der Geschichtsdidaktik, in: Historische Sozialkunde 42, 2012, 2, 3-8, hier 4 .

9 Siehe hierzu Bernd Schönemann, Geschichtsdidaktik, Geschichtskultur, Geschichtswissenschaft, in: Hilke Günther-Arndt (Hrsg.): Geschichtsdidaktik, Praxishandbuch für SI und SII, Berlin 2003, 11-22.

10 Vgl. Peter Adamski/Markus Bernhardt, Diagnostizieren - Evaluieren - Leistungen beurteilen, in: Michele Barricelli/Martin Lücke (Hrsg.): Handbuch Praxis des Geschichtsunterrichts, Bd. 1. Schwalbach/ Ts. 2012, 401-435, hier 409.

11 C. Plinius Secundus d. Ä., Naturkunde, Buch VIII, hrsg. u. übers. v. Roderich König mit Gerhard Winkler. Kempten $1976,64$.
} 
wird. Neben solchen scheinbar objektiven Beschreibungen als Naturdeutungen findet sich das Einhorn aber vor allem auch in christlichen Zusammenhängen. So wird in der Bibel vermeintlich von Einhörnern gesprochen, dessen autoritäre Kraft im Kontext der vormodernen, christianisierten Gesellschaft nicht verkannt werden darf. Im bereits erwähnten Physiologus verbindet sich das antike naturkundliche Wissen mit religiösem. Darüber hinaus werden Einhörnern aber auch verschiedene Symbole zugesprochen, in der Vormoderne zumeist religiöse, wonach das Einhorn „nach und nach immer enger mit Schlüsselfiguren und Szenen aus dem Leben Christi in Verbindung“ gebracht wurde. ${ }^{12}$ So wird mit dem Einhorn beispielsweise das Motiv der Jungfräulichkeit verbunden, welches sich eben auch im Physiologus manifestiert: „Wie wird es nun gefangen? Eine reine, schön gekleidete Jungfrau setzen sie vor ihm nieder, und es springt ihr auf den Schoß, und die Jungfrau nährt das Tier und bringt es dem König in den Palast." ${ }^{13}$ Für die vormoderne Gesellschaft war die Existenz des Einhorns absolut real und durch die Verhandlung in für die Menschen des Mittelalters relevanten Texten und Bildern allgegenwärtig. Durch ihre Autorität hat es wohl auch keinen Anlass gegeben, dies zu hinterfragen. So ist es kaum verwunderlich, dass dem Horn als markantestes Kennzeichen des Tiers sogar eine heilkräftige Wirkung zugesprochen wurde. Obwohl nicht existent, wurde ,Alicorn' (womit ebendieses Horn gemeint ist) insbesondere im Spätmittelalter zu den gefragtesten Wertgegenständen in Europa. ${ }^{14}$ Darüber hinaus wurden diese aber auch als universales Medikament gehandelt. Wie Weitbrecht anhand Rolings Aufsatz zeigt, wurden mit der Enttarnung der Einhörner als Stoßzähne eines Narwals durch Thomas Bartholin im 17. Jahrhundert erste Zweifel an der Existenz von Einhörnern laut. ${ }^{15}$ Dennoch, so Roling, „hatten Bartholins Einlassungen nicht erreichen können, daß sie von der Bildfläche der globalen Marginalen verschwanden, im Gegenteil, gerade Bartholins Bereitschaft, die eigentlichen Einhörner noch immer in einem empirischen Zwischenbereich der Semiverifikation zuverorten, mußte einen erheblichen Beitrag dazu geleistet haben, sie auch weiterhin mit einem gewissen Eigenrecht zu versehen. "16

Obwohl es also offensichtlich auch kritische Stimmen gegeben hat, so ist das Einhorn im Mittelalter ein Tier wie jedes andere und noch viel mehr: In ihm findet sich, was die vormoderne Gesellschaft beschäftigt hat. Es ist die Projektionsfläche religiöser und weltlicher Deutungen. Was insbesondere bezüglich der starken Symbolkraft des Einhorns in unserer modernen Gesellschaft mehr abwegig und abergläubisch er-

\footnotetext{
12 Lavers, Einhorn (wie Anm. 2), 57.

13 Hans Zimmermann, Einhorn, Löwe und Phoenix im Physiologus. Griechischer Text nach F. Sbordone (Rom 1936), deutsche Übersetzung von Hans Zimmermann, in: Quellen zum Weltbild des Mittelalters, Görlitz 2003, http://12koerbe.de/pan/einhorn. htm (letzter Zugriff: 26.5.2021)

14 Vgl. Lavers, Einhorn (wie Anm. 2), 77.

15 Vgl. Bernd Roling, Der Wal als Schauobjekt: Thomas Bartholin (1616-1680), die dänische Nation und das Ende der Einhörner, in: Paul J. Smith/Karl A. E. Enenkel (Hrsg.) : Zoology in Early Modern Culture. Intersections of Science, Theology, Philology and Political and Religious Education, Leiden 2014, 172-196.

16 Ebd., 191.
} 
scheint, war im Mittelalter nicht nur Teil der Welt, sondern bedeutsam. Wenn im Geschichtsunterricht nicht nur Vergangenheit kritisch gedacht werden soll, sondern auch der Umgang mit ihr in der Gegenwart in Form von historischen Erzählungen ${ }^{17}$, warum dann nicht auch das zeitliche Überdauern des Einhorns als Symbol thematisieren und dekonstruieren? Die damit in den Fokus gerückte Geschichtskultur, die alltagsweltliche Präsenz des Gegenstands, könnte insofern genutzt werden, um Kontinuität und Wandel des Einhorns in puncto Überdauern und Umdeutung zu reflektieren; ebensolche Kontingenzerfahrungen verweisen wiederum auf die prinzipielle Offenheit von Geschichte. Mit diesen Erkenntnissen werden meines Erachtens auch die Einwände, ein fiktives Wesen in den Geschichtsunterricht zu integrieren, ad absurdum geführt.

\section{Das Einhorn im Geschichtsunterricht: Ein Widerspruch?}

So sieht auch Julia Weitbrecht Potenzial für die Geschichtsdidaktik. In ihren Vorschlägen wirft sie verschiedene Schlaglichter. Als Literaturwissenschaftlerin verweist sie natürlich auf die Text- und Sprachkompetenz, die durch Fabeln, verschiedene Auslegungen und moralisches Handlungswissen in den Fokus rückt. Dem würde ich zustimmen, gerade weil die „Reflexion des Verhältnisses von Sprache(n) und historischem Lernen [...] im Horizont geschichtsdidaktischen Denkens“ nichts Neues darstellt. ${ }^{18}$ Dass auch Sprache eine wichtige Komponente des Geschichtsunterricht ist, liegt insofern auf der Hand, als historisches Verstehen und Denken als „mentale Operationen“ zunächst auch Sprachleistungen sind, die das Vermögen zu einer sprachlichen Dekonstruktion voraussetzen. ${ }^{19}$ Nicht umsonst definiert Barricelli die narrative Kompetenz als „linguistische und kognitive Kompetenz" ${ }^{20}$ So wie hier gemeint ist, dass Sprachenlernen und Fachlernen als „zwei Seiten einer Medaille, die bei der Planung und Strukturierung historischer Lehr- und Lernprozesse integral betrachtet werden müssen “21, böte sich die Wissens- und Faszinationsgeschichte des Einhorns an, um inhaltliches, kognitives und sprachliches Lernen gleichermaßen zu evozieren. ${ }^{22}$

Ein weiteres Schlaglicht wirft Julia Weitbrecht auf die bereits angerissene Frage nach der Historizität des Wissens, worin sie insbesondere für die Unterscheidung von Fakt und Fiktion Potenzial sieht. Auch an dieser Stelle möchte ich nochmal auf das Ge-

17 Vgl. Christoph Kühberger, Erinnerungskulturen als Teil des historisch-politischen Lernens, in: Informationen zur Politischen Bildung 32, 2010, 39-42, hier 39.

18 Saskia Handro, Sprache(n) und historisches Lernen. Eine Einführung, in: Zeitschrift für Geschichtsdidaktik 14, 2015, 5-24, hier 5.

19 Vgl. ebd.

20 Michele Barricelli, "The story we're going to try and tell". Zur andauernden Relevanz der narrative Kompetenz für das historische Lernen, in: Zeitschrift für Geschichtsdidaktik 7, 2008, 140-153, hier 143, zit. nach Handro, Sprache (n) und historisches Lernen (wie Anm. 18), 12.

21 Ebd.

$22 \mathrm{Vgl.} \mathrm{Handro,} \mathrm{Sprache(n)} \mathrm{und} \mathrm{historisches} \mathrm{Lernen} \mathrm{(wie} \mathrm{Anm.} \mathrm{18),} 12$. 
schichtsbewusstsein rekurrieren. So mögen die schon von Pandel in den geschichtsdidaktischen Diskurs eingebrachten Dimensionen des Geschichtsbewusstseins zeigen, wenn er bezüglich der geschichtlichen Dimension mit der zweiten Dimension auf das "Realitätsbewusstsein“, die Kategorien real-fiktiv voneinander unterscheidet, ${ }^{23}$ dass sich doch gerade durch die Konfrontation mit Fiktivem ein solches Bewusstsein erst entfalten kann. Und soll nicht sowieso ein ,historisches Problem' den Verlauf des Geschichtsunterrichts bestimmen?24 Die Antwort ist ja - denn seit den 1970er Jahren ist die Problemorientierung fester Bestandteil der Geschichtsdidaktik und fordert genau das - die ,Lösung‘ eines Problems als ursprüngliche „Antithese zu einem noch dominant chronologisch-epochal aufgebauten, faktenorientierten, darbietenden Unterrichtsstil““ ${ }^{25}$ Vielleicht kann auch die historische Auseinandersetzung mit dem Einhorn als eine solche Antithese gewertet werden. Eine Fokussierung individueller Momente des historischen Lernens knüpft daher auch an die subjektorientierte Geschichtsdidaktik an, die Schüler*innen als denkende Subjekte in den Fokus von Geschichtsdidaktik und -unterricht stellt. Eine Perspektive, die vor dem Hintergrund der Abwendung des rein deklarativen Geschichtswissens zunehmend an Wichtigkeit gewinnt und die Fähigkeit einer kritischen Haltung hervorhebt.

Neben der Auseinandersetzung mit Tierpraktiken, die im Zeitalter des Anthropozäns Themen wie Tierschutz, Vegetarismus und Co. in den Vordergrund rücken und deshalb ohne Zweifel sinnvoll für jeglichen Unterricht erscheinen, verweist Julia Weitbrecht vor allem auf das Potenzial, das sich für die Repräsentation von Tieren in historischer Perspektive ergibt. Zu nennen sind diesbezüglich Aspekte wie Herrschaftspraxis, Symbolpolitik und religiöse Differenz. Hier ergeben sich auch Möglichkeiten im Bereich der Werteerziehung, wie Weitbrecht in ihrem Vortrag einwarf, wenn sich beispielsweise durch die Dekonstruktion antisemitischer Ausdrücke wie ,Judensau' eine neue MenschTier-Relation eröffnet, die Diffamierungen an Tiervergleiche koppelt. Da die Auseinandersetzung mit Vergangenheit niemals wertfrei sein kann, kommt der Förderung der Urteilsfähigkeit des Individuums ohnehin eine wesentliche Rolle im Geschichtsunterricht zu. ${ }^{26}$ Der Umgang mit - und sprachlicher Missbrauch von - Tieren kann an dieser Stelle eine geeignete Diskussions- und Reflexionsgrundlage bieten. Demgegenüber sind wir an dieser Stelle auch mit Grenzen der Umsetzbarkeit konfrontiert: Es handelt sich hierbei nämlich um einen gemeinhin akademischen Diskurs, der nicht ,einfach so ' auf die schulische Praxis übertragen werden kann. So gilt es dahingehend, Zwischenschritte zu entwickeln.

$23 \mathrm{Vgl}$.Ammerer, Geschichtsbewusstsein (wie Anm. 8), 5.

24 Vgl. Michele Barricelli, Problemorientierung, in: Ulf Mayer/Hans-Jürgen Pandel/Gerhard Schneider (Hrsg.): Handbuch Methoden im Geschichtsunterricht, 2. Aufl. Schwalbach/Ts. 2007, 78-90, hier 78.

25 Ebd.

26 Vgl. Jochen Pahl/Christoph Wilfert, HT 2018: Werte und Werteerziehung im Geschichtsunterricht?!, 25.09.2018-28.09.2018 Münster, in: H-Soz-Kult, 30.11.2018, www.hsozkult.de/conferencereport/id/tagungsberichte-7987 (letzter Zugriff: 26.5.2021). 
Insgesamt kann man wohl behaupten, dass in der Wissens- und Faszinationsgeschichte des Einhorns, aber auch in der Auseinandersetzung mit der mittelalterlichen Tierkunde allgemein so manches Potenzial steckt, welches auf den ersten Blick wohl nicht erahnt worden wäre. Vor allem die Möglichkeit, zentrale geschichtsdidaktische Konzepte wie Geschichtsbewusstsein, Problem- und Subjektorientierung sowie Kompetenzorientierung mit dem Gegenstand Einhorn zu vereinen, sei an dieser Stelle nochmals besonders betont. Schlussendlich lohnt vielleicht noch ein Blick auf die Prinzipien guten Geschichtsunterrichts, wenn man ob der Legitimation des Gegenstands ganz sichergehen möchte: Hier ergeben sich nach Gautschi u. a. insgesamt 18 Prinzipien, die ich an dieser Stelle nicht einzeln aufführen möchte. Es sei aber darauf verwiesen, dass sich viele auf die von Julia Weitbrecht genannten Aspekte beziehen lassen. Herausgegriffen sei z. B. das zweite Prinzip, in dem es heißt, die Themen des Geschichtsunterrichts sollen „exemplarisch ausgewählte Aspekte von Schlüsselproblemen“ spiegeln und die „lokale und globale Verantwortung der Mitglieder einer Gesellschaft deutlich“ machen. ${ }^{27}$ Ebendies ist meines Erachtens genau der Knackpunkt der Materie, wenn Tierperspektiven erschlossen werden und die Welten der Gegenwart und Vergangenheit vernetzen, insbesondere, wenn diese einen Anlass geben, über aktuelle Diskurse wie Umwelt, Feminismus und Rassismus nachzudenken. Daneben stellen eben jene Tierperspektiven aber auch die Möglichkeit bereit, diese Welten voneinander abzugrenzen, weil Tiere eben keine Menschen sind, sondern bloß Mittel, diese zu spiegeln.

Natürlich muss auch erwähnt werden, dass die Beschäftigung mit dem Einhorn und anderen Tieren ebenso Probleme, insbesondere für die unterrichtliche Praxis birgt. Vor einer Herausforderung stehen Lehrkräfte, wenn sie nur auf sehr wenige aufgearbeitete Wissensbestände zurückgreifen können. Das wäre also Ausblick und zuvorderst Appell an die literaturwissenschaftliche Mediävistik und Geschichtswissenschaft, diesem Problem Abhilfe zu schaffen. Andererseits lassen sich Umstände wie diese immer auch produktiv nutzen: zu denken sei beispielsweise an die Lernform des Forschenden Lernens. Abschließend möchte ich aber das große Potential betonen, das sich aus meiner Sicht ergibt, wenn (Mensch-)Tier-Relationen dazu dienen können, Anschaulichkeit, Vielfältigkeit, Innovation und damit auch neue Denkräume in den Geschichtsunterricht zu bringen. Dieses kann sich vor allem durch jene Komponenten entfalten, die sich auf den aktuellen Zeitraum, d. h. die aktuelle Gegenwart beziehen, ${ }^{28}$ wie es beispielsweise die ,Human Animal Studies zeigen, die die Mensch-Tier-Beziehungen aus soziologischer, biologischer, geschichtswissenschaftlicher, psychologischer usw. Sicht analysieren. Damit konnte durch Julia Weitbrechts Überlegungen ein erster wichtiger Beitrag geleistet werden, den es in Zukunft gilt, in und für die Geschichtsdidaktik fruchtbar zu machen.

27 Peter Gautschi/Markus Bernhard/Ulrich Mayer, Guter Geschichtsunterricht - Prinzipien, in: Michele Barricelli/Martin Lücke (Hrsg.): Handbuch Praxis des Geschichtsunterrichts, Bd. 1, 2. Aufl. Schwalbach/Ts. 2017, 326-363, hier 345.

28 Vgl. Thomas Martin Buck, Lebenswelt- und Gegenwartsbezug. In: Michele Barricelli/Martin Lücke (wie Anm. 9), 289-301, hier 289. 


\section{Autorin}

\section{Charis-Fey Westensee}

Lehrerin im Vorbereitungsdienst, ehemalige wissenschaftliche Hilfskraft für Didaktik der Geschichte an der Christian-Albrechts-Universität zu Kiel.

chariswestensee@gmx.de 